\title{
Which Nutritional Factors Are Good for HDL?
}

\author{
Hidekatsu Yanaia, c, Norio Tada ${ }^{\mathrm{b}}$
}

\section{To the Editor}

High-density lipoprotein (HDL) is a lipoprotein which has anti-atherogenic property by reversing cholesterol transport from the peripheral tissues to liver. Low HDL-cholesterol (HDLC) as well as high LDL-C is associated with the development of coronary heart diseases $[1,2]$. Low HDL-C is commonly observed in patients with insulin resistance, obesity and type 2 diabetes. In our previous study [3], serum HDL-C in type 2 diabetes [4], especially in type 2 diabetic patients with obesity [5], was lower than young lean men [6] and low Framingham risk score subjects [7] (Fig. 1), suggesting a significant influence of obesity, type 2 diabetes and insulin resistance on serum HDL-C levels.

Abnormal lipid metabolism induced by obesity, insulin resistance and type 2 diabetes was shown in Figure 2. Insulin resistance increases activity and expression of hormone-sensitive lipase (HSL) in adipose tissue, which catalyzes the breakdown of triglyceride (TG), releasing free fatty acids (FFAs) (Fig. 2) [8]. Increased FFA entry to liver elevates hepatic production of very low-density lipoprotein (VLDL) which is a TG-rich lipoprotein. Insulin resistance also decreases the activity of lipoprotein lipase (LPL), the rate-limiting enzyme of the catabolism of TG-rich lipoproteins such as VLDL [9]. The formation of HDL is related with the catabolism of TG-rich lipoproteins by LPL [10]. Therefore, reduced LPL activity increases VLDL and reduces HDL.

Insulin resistance is associated with diminished LDLreceptor (LDL-R) [11], and intestinal mRNA expression of Niemann-Pick C1-like 1 (NPC1L1) protein is increased in diabetes [12], suggesting that insulin resistance and diabetes may increase serum LDL-C by reducing clearance and enhancing cholesterol absorption.

The dietary and nutritional factors could influence on HDL metabolism. To make "Dietary Reference Intake for Japanese 2020 (for low HDL-C)", we searched meta-analyses of randomized controlled trials (RCTs) which investigated effects of energy and carbohydrate intake [13], glycemic index and

Manuscript submitted October 4, 2018, accepted October 16, 2018

aDepartment of Internal Medicine, National Center for Global Health and Medicine Kohnodai Hospital, Chiba, Japan

${ }^{\mathrm{b}}$ The Jikei University School of Medicine, Tokyo, Japan

${ }^{\mathrm{c} C}$ Corresponding Author: Hidekatsu Yanai, Department of Internal Medicine, National Center for Global Health and Medicine Kohnodai Hospital, 1-7-1 Kohnodai, Chiba 272-8516, Japan. Email: dyanai@hospk.ncgm.go.jp

doi: https://doi.org/10.14740/jocmr3646 intake of dietary fiber [14], intake of soy and non-soy legume [15] and consumption of various fatty acids [16], on serum HDL-C levels. Seen in Figure 2, since low HDL-C is likely to accompany with obesity, insulin resistance and high TG. Elevation of LDL-C can accompany with low HDL-C; moreover, atherogenic properties of LDL such as small dense LDL, oxidized LDL and glycated LDL are observed in type 2 diabetes [17]. Therefore, we investigated effects of nutritional factors on metabolic parameters other than HDL-C such as LDL-C, TG, body weight and waist circumference.

The summary of effects of nutritional factors on HDL-C and other metabolic parameters was shown in Table 1. Low carbohydrate diet increased HDL-C and decreased TG, however, increased LDL-C. Dietary fiber consumption did not affect HDL-C and TG, however, reduced LDL-C. Soy consumption increased HDL-C and deceased both TG and LDL-C. Saturated fatty acid consumption increased HDL-C, however, also increased LDL-C. Industrially produced trans fatty acid consumption reduced HDL-C and increased LDL-C.

When we consider effects of nutritional factors on HDL$\mathrm{C}$ to prevent atherosclerosis, we should think about effects of nutritional factors on other lipids, especially LDL-C.

\section{Conflict of Interest}

The authors declare that they have no conflict of interest concerning this article.

\section{References}

1. Kitamura A, Iso H, Naito Y, Iida M, Konishi M, Folsom AR, Sato S, et al. High-density lipoprotein cholesterol and premature coronary heart disease in urban Japanese men. Circulation. 1994;89(6):2533-2539.

2. Satoh H, Nishino T, Tomita K, Saijo Y, Kishi R, Tsutsui $\mathrm{H}$. Risk factors and the incidence of coronary artery disease in young middle-aged Japanese men: results from a 10-year cohort study. Intern Med. 2006;45(5):235-239.

3. Yanai H, Hirowatari Y, Ito K, Kurosawa H, Tada N, Yoshida H. Understanding of Diabetic Dyslipidemia by Using the Anion-Exchange High Performance Liquid Chromatography Data. J Clin Med Res. 2016;8(5):424-426.

4. Yoshida H, Hirowatari Y, Kurosawa H, Manita D, Yanai $\mathrm{H}$, Ito $\mathrm{K}$, Tada $\mathrm{N}$. Estimation of lipoprotein profile in patients with type II diabetes and its relevance to remnant lipoprotein cholesterol levels. Atherosclerosis. 2012;222(2):541-544. 


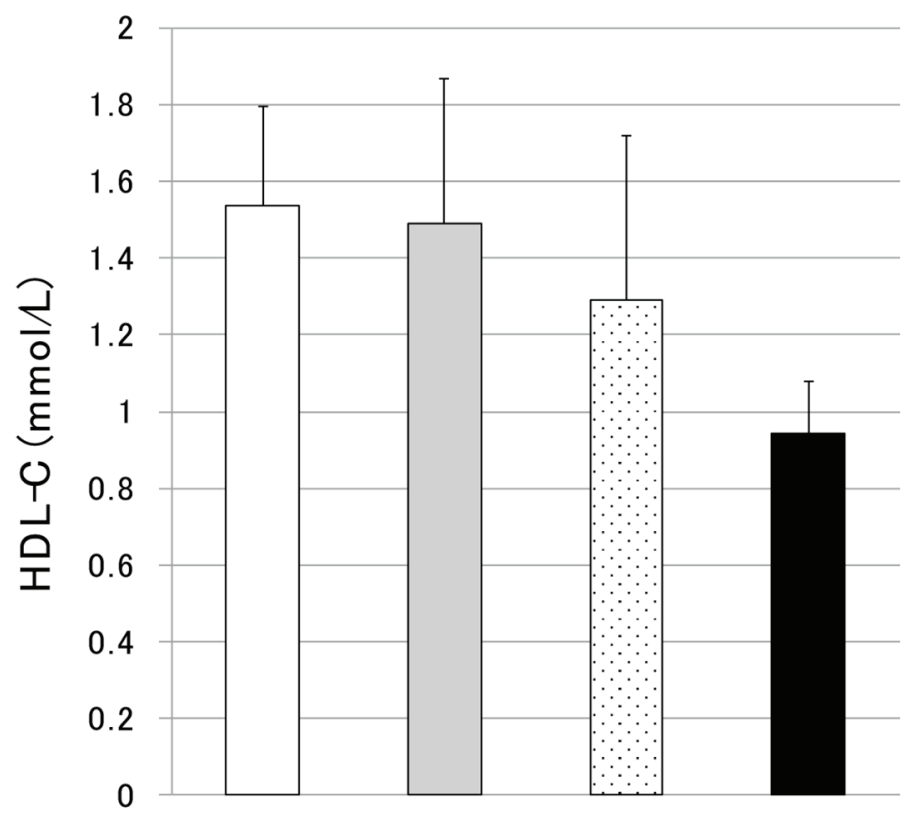

Young men [6]

Middle-aged people with low Framingham risk score [7]

Type 2 diabetic patients without obesity [4]

Type 2 diabetic patients with obesity [5]

Figure 1. Serum HDL-C levels in young men, middle-aged people with low Framingham risk score, type 2 diabetic patients without obesity and type 2 diabetic patients with obesity. This figure was made by modification of data in our previous report [3].

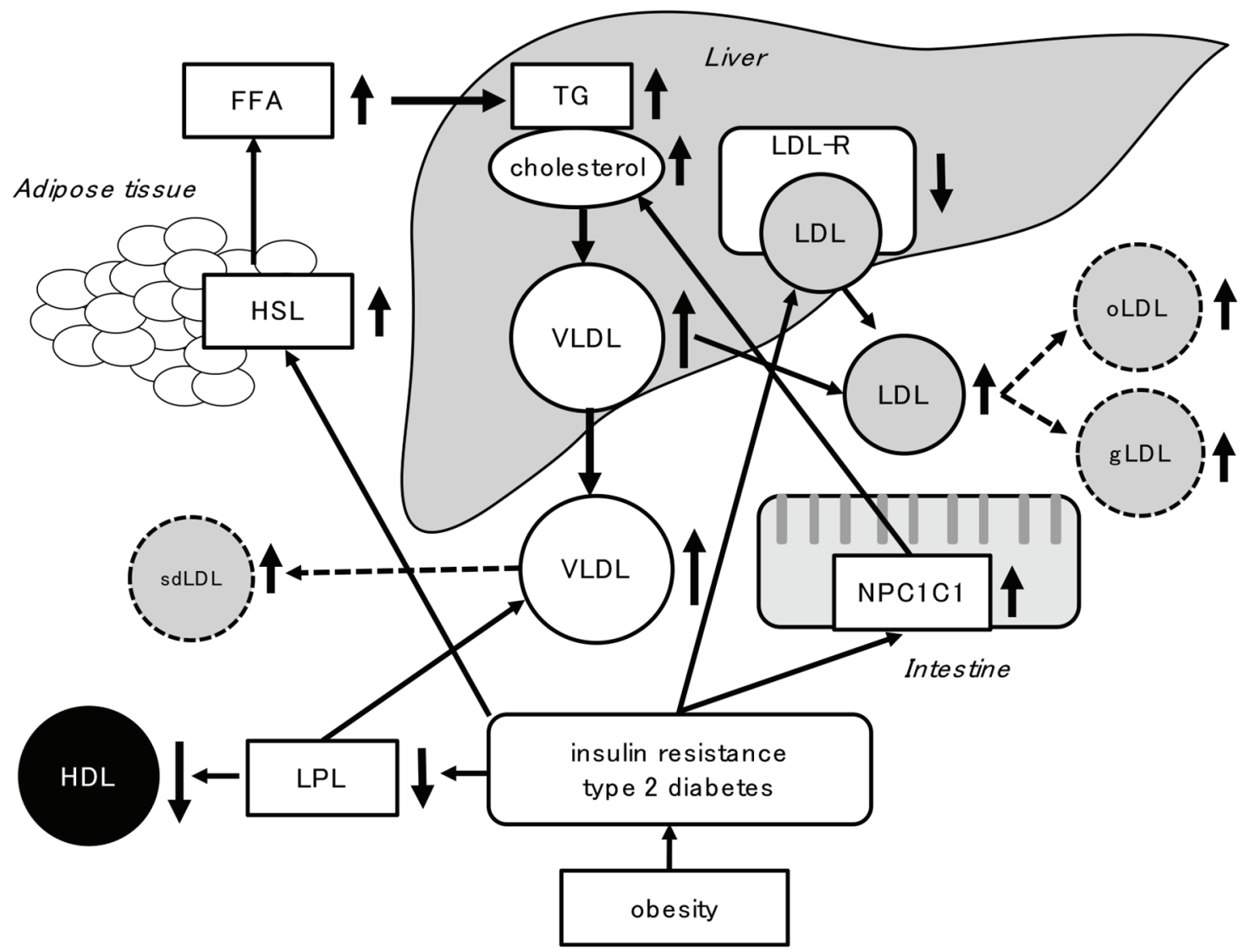

Figure 2. Abnormal lipid metabolism induced by obesity, insulin resistance and type 2 diabetes. FFA: free fatty acid; gLDL: glycated LDL: HSL: hormone-sensitive lipase; LDL-R: LDL-receptor; LPL: lipoprotein lipase; NPC1L1: Niemann-Pick C1-like 1; oLDL: oxidized LDL; sdLDL: small dense LDL; VLDL: very low-density lipoprotein. 
Table 1. Effects of Nutritional Factors on HDL-C and Other Metabolic Parameters

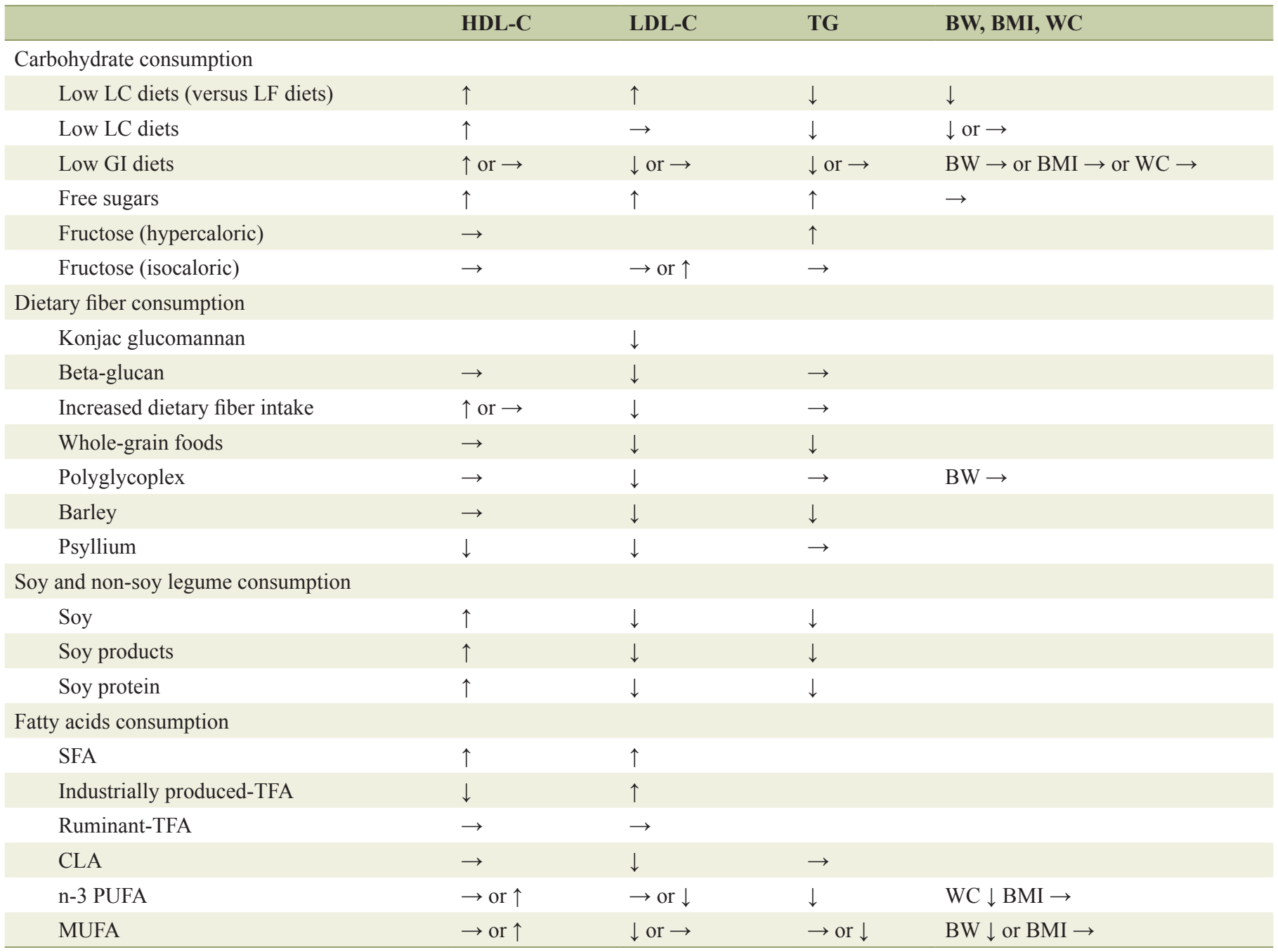

BMI: body mass index; BW: body weight; CLA: conjugated linoleic acid; GI: glycemic index; HDL-C: high-density lipoprotein-cholesterol; LC: low carbohydrate; LDL-C: low-density lipoprotein-cholesterol; LF: low fat; MUFA: mono-unsaturated fatty acid; PUFA: poly-unsaturated fatty acid; SFA: saturated fatty acid; TFA: trans fatty acid; TG: triglyceride; WC: waist circumference.

5. Yanai H, Hamasaki H, Adachi H, Moriyama S, Hirowatari Y. Effects of liraglutide, a human glucagon-like peptide-1 analog, on glucose/lipid metabolism, and adipocytokines in patients with type 2 diabetes. J Endocrinol Metab. 2011;1(3):149-151.

6. Yanai H, Yoshida H, Tomono Y, Hirowatari Y, Kurosawa H, Matsumoto A, Tada N. Effects of diacylglycerol on glucose, lipid metabolism, and plasma serotonin levels in lean Japanese. Obesity (Silver Spring). 2008;16(1):47-51.

7. Ito K, Yoshida H, Yanai H, Kurosawa H, Sato R, Manita D, Hirowatari Y, et al. Relevance of intermediate-density lipoprotein cholesterol to Framingham risk score of coronary heart disease in middle-aged men with increased non-HDL cholesterol. Int J Cardiol. 2013;168(4):38533858.

8. Sztalryd C, Kraemer FB. Regulation of hormone-sensitive lipase in streptozotocin-induced diabetic rats. Metabolism. 1995;44(11):1391-1396.
9. Nikkila EA, Huttunen JK, Ehnholm C. Postheparin plasma lipoprotein lipase and hepatic lipase in diabetes mellitus. Relationship to plasma triglyceride metabolism. Diabetes. 1977;26(1):11-21.

10. Nikkila EA, Taskinen MR, Kekki M. Relation of plasma high-density lipoprotein cholesterol to lipoprotein-lipase activity in adipose tissue and skeletal muscle of man. Atherosclerosis. 1978;29(4):497-501.

11. Roberts CK, Liang K, Barnard RJ, Kim CH, Vaziri ND. HMG-CoA reductase, cholesterol 7alpha-hydroxylase, LDL receptor, SR-B1, and ACAT in diet-induced syndrome X. Kidney Int. 2004;66(4):1503-1511.

12. Tomkin GH. The intestine as a regulator of cholesterol homeostasis in diabetes. Atheroscler Suppl. 2008;9(2):2732.

13. Yanai H, Tada N. Effects of energy and carbohydrate intake on serum high-density lipoprotein-cholesterol levels. J Endocrinol Metab. 2018;8(2-3):27-31. 
14. Yanai H, Tada N. Effects of glycemic index and intake of dietary fiber on serum HDL-cholesterol levels. J Endocrinol Metab. 2018;8(4):57-61.

15. Yanai H, Tada N. Effects of intake of soy and non-soy legume on serum HDL- cholesterol levels. J Endocrinol Metab. 2018;8(4):57-61.
16. Yanai H, Tada N. Effects of consumption of various fatty acids on serum HDL- cholesterol levels. J Endocrinol Metab. 2018 (in press).

17. Verges B. Lipid modification in type 2 diabetes: the role of LDL and HDL. Fundam Clin Pharmacol. 2009;23(6):681685 . 